\title{
APPROACHES TO DETERMINING THE ATTENUATION OF ELECTROMAGNETIC WAVES FROM THE CENTIMETER RANGE IN DIELECTRIC COMPOSITE MATERIALS
}

\author{
Nikolay L. Gueorguiev ${ }^{1 *}$, Aleksander N. Semenov ${ }^{2}$ \\ AND Sergey I. IVASHOV ${ }^{2}$ \\ ${ }^{1}$ Institute of Metal Science, Equipment and Technologies \\ with Hydro- and Aerodynamic Centre "Acad. Angel Balevski", \\ Bulgarian Academy of Sciences, \\ 67, Shipchenski Prohod Blvd, 1574 Sofia, Bulgaria, \\ e-mail: niki0611@abv.bg \\ ${ }^{2}$ Bauman Moscow State Technical University, \\ 5, 2-nd Baumanskaya, 105005 Moscow, Russia, \\ e-mails: semenov.an@bmstu.ru; sivashov@rslab.ru
}

\begin{abstract}
The present paper is aimed at studying the possibility of applying the technologies of non-destructive testing of different materials by means of radar methods. The main parameter that predetermines these capabilities is the attenuation of the highfrequency signal in the specific type of material. In practically significant cases its determination is possible only by experimental methods. The attenuation coefficient of ultrahigh frequency oscillations of the K-band is studied for four types of test specimens used by Bulgarian organizations working in the field of aerospace industry. Tests are performed through a specially designed test bench and laboratory installations.

Keywords: attenuation, ultrahigh frequency oscillations, K-band, test bench, laboratory installations.
\end{abstract}

\section{INTRODUCTION}

Currently, a number of organizations and companies are developing new technologies of non-destructive testing of different materials through the use and further development of radar methods. Their application depends on the type of materials subjected to this type of non-destructive testing and on the

${ }^{*}$ Corresponding author.

DOI: 10.7546/EngSci.LVIII.21.03.07 
operating frequency of the radar used. The dominant factor for the application of a radar method of non-destructive testing of different materials is the attenuation of the ultrahigh frequency signal in the specific type of material. At this stage, there is no possibility of relatively accurate determining this attenuation in real multi-structural materials, therefore, experimental methods are used for this purpose.

One of the important directions in this field is related to the study of the attenuation of ultrahigh-frequency radiation in the gigahertz range when passing through materials that are used or could be used by Bulgarian companies and organizations working in the aerospace industry. In the general case, it is possible to use two approaches: (i) direct measurement of the attenuation by a special test bench, and (ii) its indirect determination by estimating the dielectric parameters of the samples and calculating the attenuation.

To implement these approaches, samples of materials used in the construction of light aircraft were made and real tests were conducted through a specially designed test bench and laboratory installations.

\section{STATE OF THE TASK}

\subsection{Test samples}

In Institute of Metal Science, Equipment and Technologies with Hydroand Aerodynamics Centre "Acad. A. Balevski" at the Bulgarian Academy of Sciences (IMSETHC-BAS) 38 standard samples of materials have been made, which are used or could be used by Bulgarian companies and organizations working in the field of aerospace industry. For the study four characteristic samples were selected, as follows:

- Test sample TS 1.4.1 represents two plates with seven layers of fiberglass $(110+160+3 \times 280+160+110) \mathrm{g} / \mathrm{m}^{2}$ with dimensions $465 \times 260 \times 2 \mathrm{~mm}$, Fig. 1;

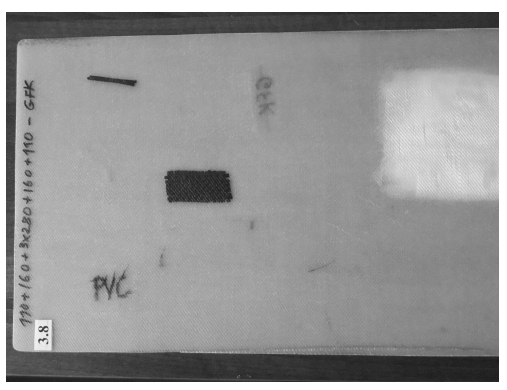

Fig. 1. TS 1.4.1

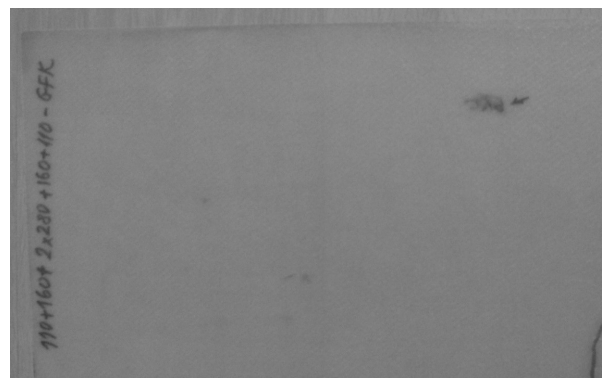

Fig. 2. TS 1.4 .2

Engineering Sciences, LVIII, 2021, No. 3 


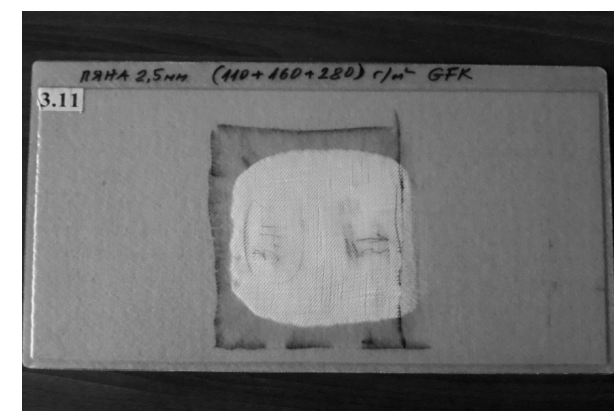

Fig. 3. TS 2.10

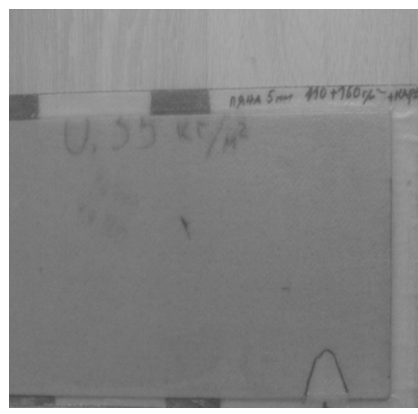

Fig. 4. TS 2.12

- Test sample TS 1.4.2 represents two plates with six layers of fiberglass $(110+160+2 \times 280+160+110) \mathrm{g} / \mathrm{m}^{2}$ with dimensions $465 \times 260 \times 1 \mathrm{~mm}$, Fig. 2.

- Test sample TS 2.10 is a plate of foam with dimensions $405 \times 210 \times 3.33 \mathrm{~mm}$ and marked defect reinforced with three layers of fiberglass $(110+160+280)$ $\mathrm{g} / \mathrm{m}^{2}$, a total of four layers, Fig. 3 ;

- Test sample TS 2.12 is a plate of foam with dimensions $395 \times 210 \times 6.02 \mathrm{~mm}$ and marked defect (crack and non-stick area) reinforced with two layers of fiberglass $(110+160) \mathrm{g} / \mathrm{m}^{2}$ plus carbon with a paper component (back), a total of four layers, Fig. 4.

\subsection{Test bench}

To determine the attenuation of microwave oscillation with a frequency of $24 \mathrm{GHz}$ during its propagation in the studied materials, a test bench was developed in IMSETHC-BAS shown in Fig. 5 [1]. It consists of a pedestal and

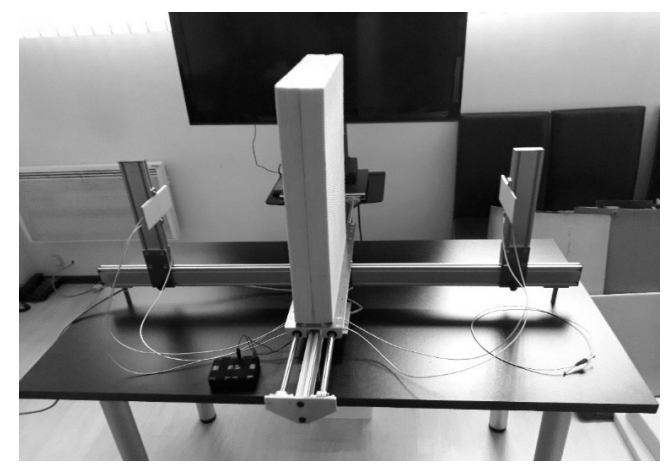

Fig. 5. Test bench 
a frame, on both sides of which antenna blocks are located. Each antenna unit includes one microwave transmitting antenna and two microwave receiving antennas. A mobile platform is installed between the antenna blocks, on which the tested sample is placed, and an electronic block is installed under the mobile platform. The information is processed by means of specialized software installed in a laptop, and the results can be displayed on an external monitor. To reduce external influences and parasitic radiation, the entire installation is covered with radio-absorbing material, Fig. 6.
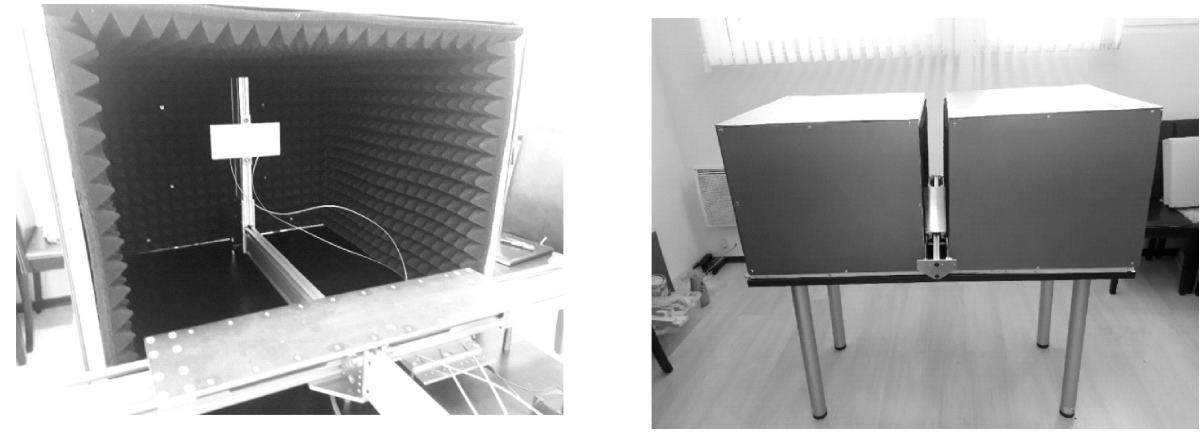

Fig. 6. Test bench covered with radio-absorbing material

\subsection{Laboratory installations}

Measurement of the complex value of the dielectric constant $\varepsilon$ at radio frequencies is important in many fields of science and technologies, and in particular to determine the attenuation coefficient of the high-frequency oscillations passing through them. Modern Vector Network Analyzers (VNAs) make it possible to simplify experiments, and on the basis of the system parameters to obtain data on the reflection and transmission coefficients in the studied materials, and then to estimate the complex dielectric constant.

To assess the dielectric constant and the tangent of the dielectric loss angle in the Test Sample (TS), the method of estimating the transmission and reflection coefficient in the waveguide (Transmission/Reflection Line method, TRL) was chosen [2-3]. In line with this method, a waveguide insert is placed between calibrated waveguide connectors filled with a dielectric, and the system parameters $S_{11}$ and $S_{21}$ are estimated. Then the coefficients of reflection $\Gamma$ and transmission $T$ are calculated, on the basis of which estimates $\varepsilon=\varepsilon^{\prime}-j \varepsilon^{\prime}$ are obtained by means of recalculation methods.

The considered method is suitable for waveguides fully filled with dielectric material. However, for samples where there is no way to fill in the waveguide, 
it is proposed to use a modification of this method, which consists in solving optimization problem of finding material parameters based on experimental data according to the scheme shown in Fig. 7.

Measurement method

Evaluation method

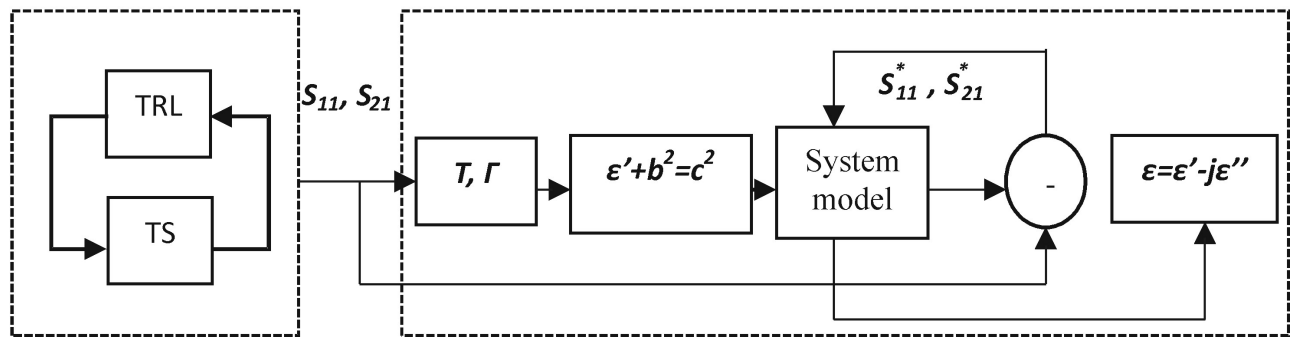

Fig. 7. TRL scheme of the modified method for complex estimation of dielectric constant

The experimental data were recorded in Bauman Moscow State Technical University (BMSTU) on a Rohde \& Schwarz ZVA24 vector network analyzer in the K-frequency range (short-wave part of the centimeter range). In the measurements, an insert of a rectangular waveguide with a cross-sectional area of $7.2 \times 3.4 \mathrm{~mm}$ and a length of $85 \mathrm{~mm}$ was used, inside which the TS was placed. Figure 8 shows a photograph of a layout for measuring $S$ parameters.

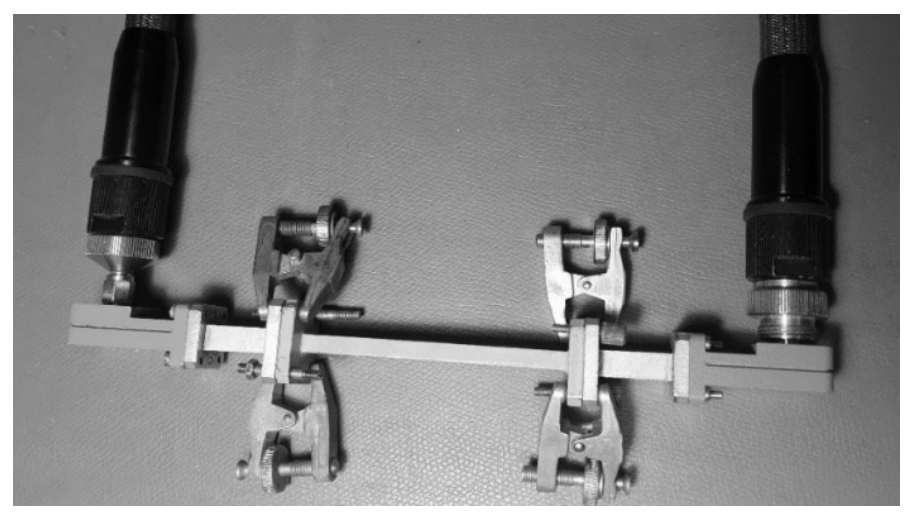

Fig. 8. Model of measuring $S$ parameters of the system

For research, dielectric inserts are cut from the test samples into a waveguide and electrodynamic models of the system are compiled in ANSYS Electromagnetics Suite 2020, the parameters of which have been optimized for the desired value of the complex dielectric constant according to the criterion of 
minimum root-mean-square deviation of the measured S-parameters compared to the model ones. The electrodynamic models consist of a waveguide model and a test sample model. The latter varies depending on the number of layers in the test sample, i.e. one layer of fiberglass for TS 1.4.1 and TS 1.4.2, two layers (fiberglass and foam) for the TS 2.1.2, and three layers (fiberglass, foam and fiberglass) for TS 2.10. All test sample models are placed along the wide side of the waveguide. Each layer in models has a variable permittivity and a loss tangent. The model system parameters $S_{11}$ and $S_{21}$ are calculated by means of Finite element method. The difference between experimental system parameters and modeled ones causes a change in the permittivity and the loss tangent until the minimum of RMS is not reached.

In addition, a laboratory test is performed in IMSETHC-BAS in order to determine the attenuation of oscillation with the frequency of $3.5 \mathrm{GHz}$ when passing through TS 2.12. For this purpose the waveguide measuring line P1-7 is used, Fig. 9.
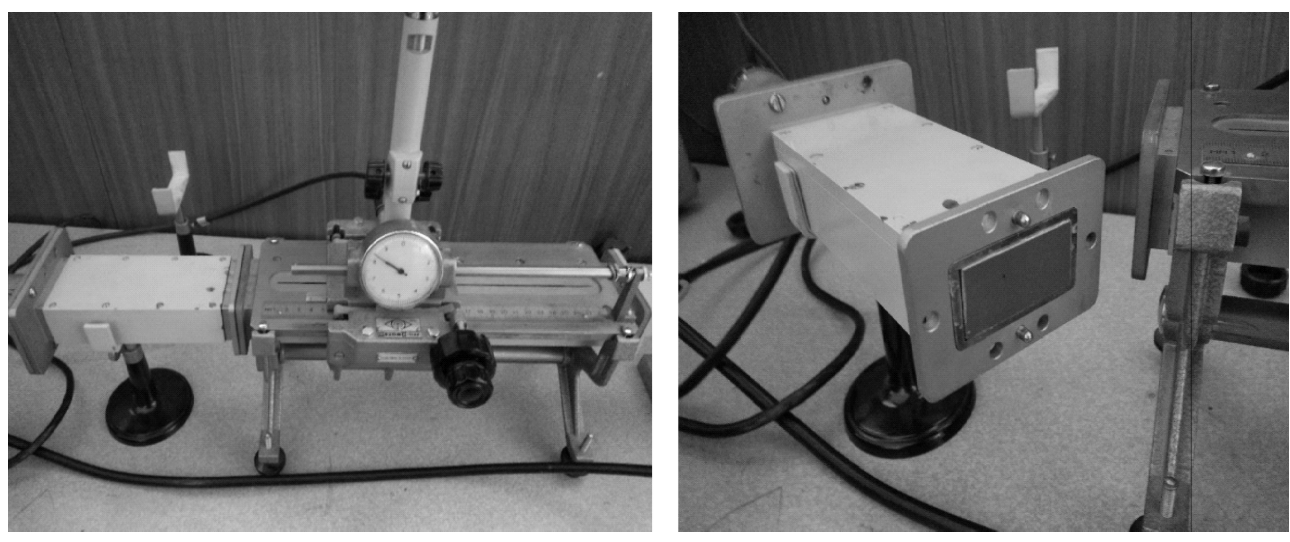

Fig. 9. Waveguide measuring line P1-7 general type and with test sample

The determination of the attenuation coefficients of the test specimens is performed through the test bench in accordance with the developed methodology. The measurement technology is a process of sequential transmission of microwave oscillations from the transmitter connected to one antenna unit, as the receiving antennas of the opposite antenna unit receive the signals passed through the test pattern, after which the transmitter connected to the second antenna unit emits microwave oscillations and the first one receives them. 


\section{TEST RESULTS}

The tests are performed by the test bench in 20 consecutive cycles, determining the maximum, minimum and average values of the attenuation coefficient. The permissible difference between the results of different tests for one sample is $5 \%$. The test results obtained by means of the test bench are given in Table 1.

To determine the attenuation coefficients of the specimens tested by the laboratory set-up it is accepted that an estimate of the attenuation coefficient $\alpha$ of a plane wave in a dielectric with losses and $\mu \approx 1$ can be obtained by the ratio:

$$
\alpha=\Im m\left[\frac{2 \pi}{\lambda} \sqrt{\varepsilon^{\prime}(1-j \operatorname{tg}(\delta))}\right]
$$

where $\Im m[$.$] is the imaginary part of expression.$

Table 1. Attenuation coefficients determined by the test bench

\begin{tabular}{|c|c|c|c|c|}
\hline Sample & TS 2.12 & TS 2.10 & TS 1.4.1 & TS 1.4.2 \\
\hline Attenuation coefficient - average value & 0.88 & 0.83 & 0.78 & 0.79 \\
\hline
\end{tabular}

In essence, the use of the laboratory set-up is associated with the acceptance of a given test sample as a homogeneous dielectric, despite the fact that it is in fact a multilayer composite material composed of components of different composition. This approach is applicable to materials with relatively low attenuation and does not take into account the reflection and refractive indices of the ultrahigh frequency oscillations.

Sample TS 2.12 is approximately $6.1 \mathrm{~mm}$ thick, and it is inserted into a waveguide of approximately half thickness from two layers: fiberglass $(0.3 \mathrm{~mm}$ thick) and foam $(3.1 \mathrm{~mm})$ - a total of $3.4 \mathrm{~mm}$ thick. The measurement results are for the foam and for the glass fiber parameters the results are taken from previous evaluations of sample TS 1.4.2.

For sample TS 2.10 with a thickness of $3.33 \mathrm{~mm}$, an insert is made into the waveguide without reducing the thickness of three layers: glass fiber $(0.3 \mathrm{~mm}$ thick), foam $(2.7 \mathrm{~mm})$, and glass fiber $(0.3 \mathrm{~mm})$. The measurement result also applies to the foam, and the glass fiber parameters are taken from the results of previous evaluations of sample TS 1.4.2.

To calculate the attenuation coefficient through TS, the sequence of layers is generally taken into account (without taking into account multiple reflections of the layer boundaries).

The values of $\varepsilon^{\prime}, \operatorname{tg}(\delta)$, and attenuation coefficient $\alpha$ of TS 2.12 determined by the laboratory installation of IMSETHC-BAS are given in Table 2, and 
Table 2. Values of $\varepsilon^{\prime}, \operatorname{tg}(\delta)$, and attenuation coefficient $\alpha$ of TS 2.12 determined by the laboratory installation of IMSETHC-BAS

\begin{tabular}{|c|c|}
\hline & TS 2.12 \\
\hline$\varepsilon^{\prime}$ & 1.1566 \\
\hline $\operatorname{tg}(\delta)$ & 0.0092 \\
\hline Loss factor & 0.998 \\
\hline Attenuation coefficient $\alpha$ & $0.36257 \mathrm{~m}^{-1}$ \\
\hline Attenuation coefficient $\alpha, \mathrm{dB}$ & $-3.15 \mathrm{~dB} \cdot \mathrm{m}^{-1}$ \\
\hline
\end{tabular}

the resulting estimates of the complex permittivity determined by BMSTU's laboratory installation are given in Table 3 . Table 3 shows that TS 2.12 despite being almost twice as thick but reinforced with two layers of fiberglass causes about $15 \mathrm{~dB} \mathrm{~m}^{-1}$ less attenuation than TS 2.10, which is reinforced with three layers of fiberglass, two of which are like TS 2.12, but the third reinforcing layer has a much higher density $-280 \mathrm{~g} / \mathrm{m}^{2}$.

In Tables 2 and 3 it can be seen that the attenuation in TS 2.12 increases with increasing the signal frequency, which corresponds to the well-known dependencies for the non-resonant attenuation of different microwave oscillations in a given medium.

Table 3 shows that TS 1.4 .1 again causes at least $14.85 \mathrm{~dB} \mathrm{~m}^{-1}$ higher attenuation than TS 1.4.2 due to the fact that there are additional reinforcing layers of high density fiberglass - from $280 \mathrm{~g} / \mathrm{m}^{2}$. From this it can be concluded that a layer of fiberglass with a density of $280 \mathrm{~g} / \mathrm{m}^{2}$ leads to the attenuation approximately $15 \mathrm{~dB} \mathrm{~m}^{-1}$.

The results obtained through direct measurements on the test stand and those obtained by means of the indirect laboratory method are given in Tables 2 and 3, respectively, which vary according to the same dependence for the individual samples. At the same time, their specific values differ by up to $25 \%$, which is explained by the accepted simplifications and approximations in the laboratory method.

\section{CONCLUSIONS}

The conducted researches demonstrate the reliability of the results obtained in different ways and the applicability of the used test bench and laboratory installations of determining the attenuation of microwave oscillations when passing through different materials.

All tested samples have a low absorption close to the measurement accuracy limit of laboratory installations. Therefore, it is advisable to conduct a test 


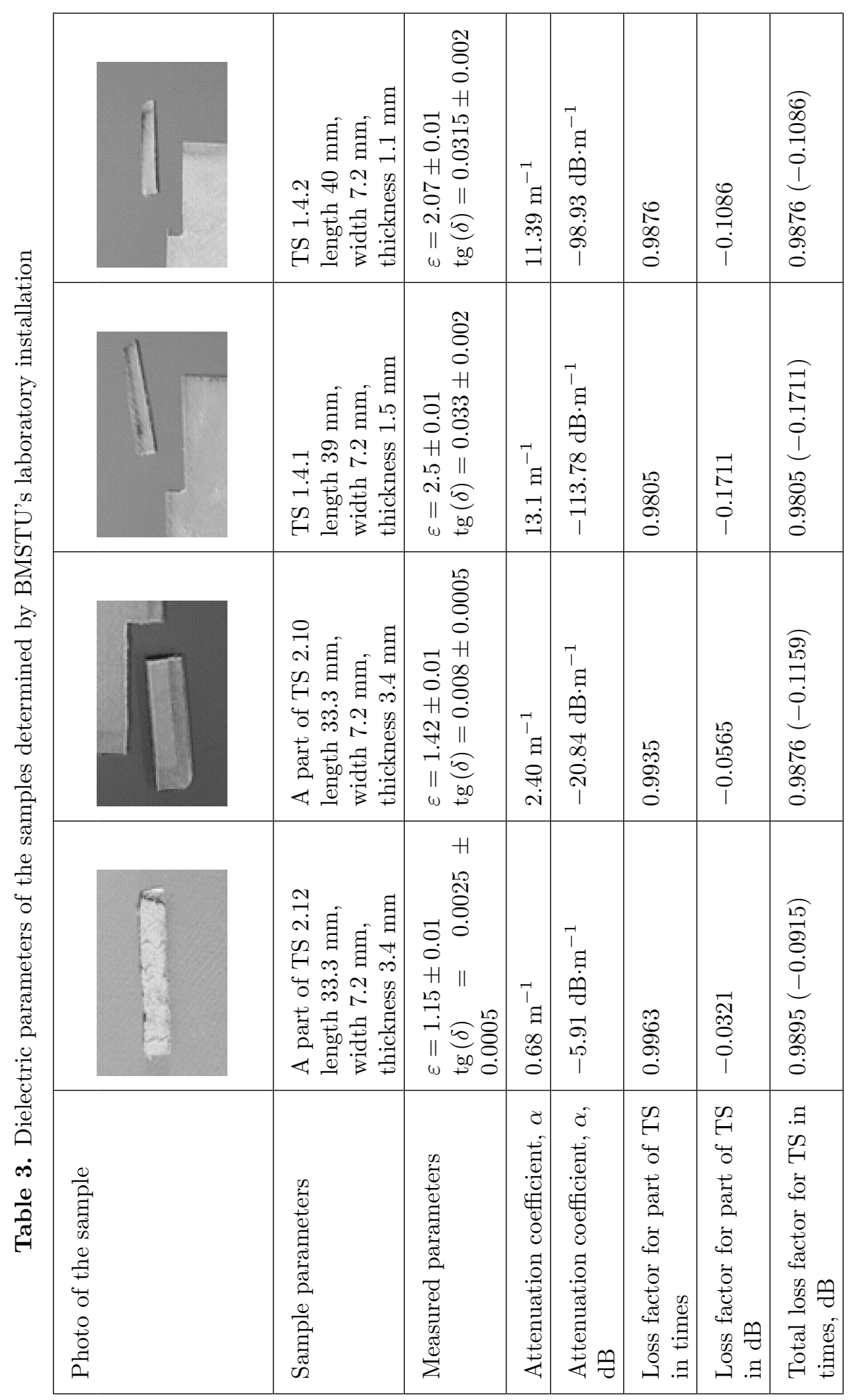


with several samples located one behind the other in the respective waveguide.

The results prove that the tested samples used by Bulgarian organizations working in the aviation industry allow non-destructive testing by radar methods in the K-band, where the accuracy and resolution are very good.

Study also shows that the use of the test specimens for the construction of hulls and other parts of aircraft and other bodies does not interfere with the installation and operation of different navigation, communication, sensor, information and other devices operating in the centimeter range.

The results of the study will be used in the Work package 2. Intelligent security systems, Project BG05M2OP001-1.002-0006 - Creation and Development of a Center of Competence "Quantum Communication, Intelligent Security Systems and Risk Management" (Quasar), founded by the European Regional Development Fund through the Operational programme "Science and education for smart growth".

As the tests were carried out only on certain areas of the material cut from the main sample, the variation in the absorption level was not measured. If necessary, it is appropriate to perform the tests through the laboratory installations on several (five to ten) samples from different parts of the same test sample.

\section{ACKNOWLEDGEMENTS}

The results of the study were achieved in the implementation of administrative contract No. KP-06-RUSSIA /22 dated September 28, 2019 and RFBR grant \# 19-57-18001 \19, for financing a research project with incl. No. NSF 93 with the theme "New Technologies for NDT of composites used in the aerospace industry" designated based financing conducted by Fund "Science Competition for projects under programs of bilateral cooperation in 2018 Bulgaria - Russia 2018-2019". Partly experiments were supported by the Russian Science Foundation under project \# 21-19-00043.

\section{REFERENCES}

[1] N. Lichkov, V. Pehlivanski, N. Lyaskov, and N. Penev, Method for Experimental Measurement of Attenuation of UHV Radiation during Its Propagation in a Specific Environment, in: XVIII International Scientific Congress Machines, Technologies, Materials, 2021, 75-78, ISSN 2535-0021.

[2] A-H. Boughriet, C. Legrand, and A. Chapoton, Noniterative Stable Transmission/Reflection Method for Low-loss Material Complex Permittivity Determi- 
nation, IEEE Transactions on Microwave Theory and Techniques (1997) 1 (45) $52-57$.

[3] Measurement of Dielectric Material Properties (Electronic resource), URL: https://www.rohde-schwarz.com/ru/applications/measurement-of-dielectricmaterial-propertiesapplication-note_56280-15697.html (date of access: 31.05.2021).

Received June 21, 2021 